\title{
Early- and late anthracycline-induced cardiac dysfunction: echocardiographic characterization and response to heart failure therapy
}

Janine A. M. Kamphuis ${ }^{1 *}$, Marijke Linschoten ${ }^{1}$, Maarten J. Cramer ${ }^{1}$, Pieter A. Doevendans ${ }^{1,2,3}$,

Folkert W. Asselbergs ${ }^{1,4,5}$ and Arco J. Teske ${ }^{1}$

\begin{abstract}
Background: Anthracycline-induced cardiac dysfunction (ACD) is a notorious side effect of anticancer treatment. It has been described as a phenomenon of a continuous progressive decline of cardiac function, eventually leading to dilated cardiomyopathy (DCM). This progressive nature suggests that patients with a delayed ACD diagnosis have greater compromise of cardiac function and more adverse remodeling, with a poor response to heart failure $(\mathrm{HF})$ treatment. This study aimed to delineate the impact of a delayed ACD diagnosis on echocardiographic characteristics and response to $\mathrm{HF}$ treatment.

Methods and results: From the population of our cardio-oncology outpatient clinic, 92 ACD patients were included in this study (age $51.6 \pm 16.2$ years, median cumulative anthracycline dose 329 [200-329] mg/m²), and a median follow-up of 25.0 [9.6-37.2] months after ACD diagnosis. Median time to ACD diagnosis for patients diagnosed early ( $<1$ year) and late ( $>1$ year) was 4.0 vs. 47.7 months respectively. There were no echocardiographic differences between patients diagnosed early vs. late (LVEF $43.6 \pm 4.9 \%$ vs. $43.0 \pm 6.2 \%$ and iEDV 63.6 vs. $62.9 \mathrm{~mL}$ / $\mathrm{m}^{2}$ ). Eighty-three percent of patients presented with mild LV dysfunction and in $79 \%$ the LV was not dilated. Patients diagnosed early were more likely to have (partial) recovery of cardiac function upon HF treatment initiation $(p=0.015)$.

Conclusions: In the setting of a cardio-oncology outpatient clinic, patients with ACD presented with a hypokinetic non-dilated cardiomyopathy, rather than typical DCM. Timing of ACD diagnosis did not impact HF disease severity. However, in patients receiving an early diagnosis, cardiac function was more likely to recover upon HF treatment.
\end{abstract}

Keywords: Heart failure, Anthracyclines, Cardiac dysfunction, Cardiac effects of cancer treatment

\footnotetext{
* Correspondence: j.a.m.kamphuis-4@umcutrecht.nl

'Department of Cardiology, Division of Heart and Lungs, University Medical

Center Utrecht, University of Utrecht, E03.511, PO Box 85500, 3508 GA Utrecht, The Netherlands

Full list of author information is available at the end of the article
}

(C) The Author(s). 2020 Open Access This article is licensed under a Creative Commons Attribution 4.0 International License, which permits use, sharing, adaptation, distribution and reproduction in any medium or format, as long as you give appropriate credit to the original author(s) and the source, provide a link to the Creative Commons licence, and indicate if changes were made. The images or other third party material in this article are included in the article's Creative Commons licence, unless indicated otherwise in a credit line to the material. If material is not included in the article's Creative Commons licence and your intended use is not permitted by statutory regulation or exceeds the permitted use, you will need to obtain permission directly from the copyright holder. To view a copy of this licence, visit http://creativecommons.org/licenses/by/4.0/ The Creative Commons Public Domain Dedication waiver (http://creativecommons.org/publicdomain/zero/1.0/) applies to the data made available in this article, unless otherwise stated in a credit line to the data. 


\section{Introduction}

Anthracyclines are potent antineoplastic drugs that constitute a cornerstone in the treatment of sarcomas, breast cancer and hematological malignancies. Shortly after the introduction of these agents in the 1960's, cardiac dysfunction was discovered to be an important dose-limiting side effect [1]. However, despite dosage restrictions, the incidence of anthracycline-induced cardiac dysfunction (ACD) has been found to be $6 \%$ for overt heart failure and up to $18 \%$ for subclinical cardiac dysfunction [2]. The prognosis of ACD is poor, with cardiovascular mortality rates ranging from $9 \%$ at 5 - and $24 \%$ at 10 -years [3], up to more dramatic outcomes of $60 \%$ at 2 -years in patients that have developed symptomatic HF [4].

ACD has often been classified to occur either "early" or "late", with the first subtype developing within the 1st year after treatment and the latter more than 1 year after anthracycline-containing therapy [5]. In recent years, this subdivision has however been questioned, since a release of troponins can be detected during- or early after anthracycline administration, indicating direct damage to the myocardium upon infusion $[6,7]$. The initial damage, resulting in a declined pumping capacity, will not coincide with the development of clinical manifestations of HF in a majority of patients. However, if serial echocardiographic assessment is performed, almost all asymptomatic declines are detected within the first year after anthracycline-containing chemotherapy [8]. The delayed onset of symptomatic HF may be related to the activation of compensatory mechanisms that modulate left ventricular (LV) function within a range, such that the functional capacity is preserved or only minimally depressed [9]. These compensatory mechanisms include the activation of neurohumoral systems and adaptive changes within the myocardium commonly referred to as $\mathrm{LV}$ remodeling. However, prolonged activation of these compensatory mechanisms ultimately has detrimental effects on the heart, leading to adverse LV remodeling with dilatation and wall thinning [10]. The onset of these changes typically marks the transition from asymptomaticto symptomatic HF. Based on this theory, it can be postulated that over time, patients with $\mathrm{ACD}$ develop progressive systolic dysfunction with dilated compartments, as is the case in patients with familial DCM, long lasting left-sided valvular disease or hypertension. The aim of this consecutive cohort study was to (1) evaluate the impact of a delayed diagnosis (> 1 year after anthracycline containing treatment) on echocardiographic characteristics and (2) assess the influence of timing of diagnosis on HF treatment response.

\section{Methods}

\section{Study population}

In April 2015, a cardio-oncology clinic was launched at the University Medical Center Utrecht, the Netherlands, of which the protocol has been described in detail previously [11].

In short, the patient population mainly consists of patients with breast cancer or hematological malignancies who are deemed to be at increased risk for ACD due to treatment- or patient-related factors. Furthermore, cancer patients and survivors that have not received cardiac surveillance in the past (e.g. received potentially cardiotoxic treatment prior to the initiation of the clinic in 2015), are referred for screening of long-term cardiovascular complications at the discretion of the treating (hemato-)oncologist. One of the primary aims of this clinic is to facilitate a timely diagnosis of cancer therapyrelated cardiac dysfunction (CTRCD) by performing serial echocardiographic assessments. When patients are diagnosed with CTRCD, guideline-based HF therapy [12] is initiated if there are signs or symptoms of $\mathrm{HF}$, or when the LV ejection fraction (LVEF) reaches $<45 \%$ regardless of the presence of cardiac complaints [11].

All patients referred to the cardio-oncology clinic between April 2015 and February 2019 who were treated with anthracyclines were identified. Subsequently, we selected patients that showed impaired LV function on echocardiography or cardiac magnetic resonance (CMR). We excluded patients with LV dysfunction on multiplegated acquisition (MUGA) scans, with echocardiographic images of insufficient quality, and patient that had received treatment with the cardiotoxic monoclonal antibody trastuzumab. Time between the initiation of anthracycline-containing therapy and ACD diagnosis was used to divide patients in two groups, namely early( $<1$ year) and late ( $>1$ year) ACD diagnosis, as described in the ESC Position Paper on Cancer Treatment and Cardiovascular Toxicity [5]. The study was exempted from formal approval by the Medical Ethics Committee.

\section{Definition of ACD}

Patients were diagnosed with ACD if they met one of the following two criteria: (1) LVEF decline of $\geq 10$ percentage points below the lower limit of normal (LLN) $(<$ $53 \%)$ from baseline according to the European Association of Cardiovascular Imaging (EACVI) [13], or (2) an LVEF $<50 \%$ measured at $>1$ time-point in case a baseline LVEF measurement prior to anthracycline treatment was not available. The diagnosis of ACD did not rely on the presence of symptoms or signs of heart failure and therefore, the selected population composes both patients with clinical and subclinical ACD.

Other possible causes of LV dysfunction were evaluated to determine the likelihood of ACD. Based on this assessment, patients were subdivided into three groups namely, 'definite ACD', 'ACD with concomitant heart disease' and 'possible ACD'. In patients with definite ACD, alternative causes of LV dysfunction were ruled 
out or deemed highly unlikely. For patients that did not undergo ischemia detection, ischemic heart disease was determined unlikely in the absence of chest pain, low cardiovascular risk profile ( $<2$ risk factors), no coronary artery disease (CAD) on computed tomography (CT) of the chest and lack of regional wall motion abnormalities on echocardiography. Patients were diagnosed with ACD and concomitant heart disease if other causes affected LV function, but these abnormalities were determined to be insufficient to explain the degree of LV dysfunction as a whole. Lastly, in patients with 'possible ACD', severe valvular heart disease, left bundle branch block (LBBB), sepsis-induced cardiac dysfunction and tachycardiomyopathy were ruled out. However, in these patients, the presence of ischemic heart disease had not formally been excluded while there were signs of CAD on the chest CT. None of these patients had reported any chest complaints. The diagnosis of ACD was verified by two authors (JK and ML).

\section{Oncological treatment}

We determined the timing of first anthracycline dose, as well as the total cumulative dose $\left(\mathrm{mg} / \mathrm{m}^{2}\right)$ which was calculated to an equivalent doxorubicin dose [14]. Moreover, data on mediastinal- or left-sided radiotherapy was collected.

\section{Echocardiographic analysis}

Echocardiographs were performed by trained technicians at our cardio-oncology program. An extensive analysis was performed on the echocardiogram on which $\mathrm{ACD}$ had been diagnosed and on the most recent echocardiogram. All measurements were analyzed by JK, and verified by AT. Reference values and international echocardiography guidelines for echocardiographic examination can be found in Supplemental Table 1. LV volumes and -ejection fractions were preferably determined on 3D echocardiographic images. If 3D images were not available, 2D biplane (modified Simpson's) algorithm was performed on the 4- and 2-chamber apical view. Global longitudinal strain (GLS) measurements were performed with the vendor's software package using the 4-, 3-, and 2-chamber apical view.

\section{Follow-up}

Patients with a follow-up of $\geq 6$ months, or patients with complete recovery of the LVEF within 6 months, were included in the analysis of ACD reversibility. Reversibility was based on the change between the nadir- and last LVEF measurement and was classified according to the EACVI Expert Consensus [13]. In patients lacking a baseline LVEF measurements $(n=74)$, we deemed ACD to be 1) reversible in case there was an LVEF improvement of $\geq 10$ percentage points to above the LLN, 2) partially reversible if LVEF improved 5-10 percentage points to above the LLN, and 3) irreversible if the LVEF improved $<10$ percentage points from the nadir and remained below the LLN.

\section{Statistical analysis}

Continuous data are expressed as means and standard deviations (SD) or medians and interquartile ranges [IQR]. Categorical variables are expressed as numbers (percentages). Continuous data were compared using the independent Student's $t$-test or Mann-Whitney U. Categorical data were tested using Chi-square or Fisher exact test as appropriate. Correlation was calculated with either Pearson or Spearman correlation, where appropriate. Differences between $>2$ groups were calculated using one-way analysis of variance (ANOVA) with Bonferroni post correction for multiple comparisons or the Kruskal-Wallis test. A two-sided $p$-value $<0.05$ was considered significant. Statistical analyses were performed using SPSS Statistics, version 25 (IBM Corp., Armonk, NY, USA).

\section{Results \\ Study population}

Between April 2015 to February 2019, a total of 512 patients had been evaluated at the cardio-oncology outpatient clinic (Fig. 1). Anthracyclines were administered in 342 patients, of which 44 patients were not eligible for this study due to concomitant treatment with trastuzumab. Of the remaining 298 patients, 107 (35.9\%) had LV dysfunction. In six patients, the underlying cause for the impaired LV dysfunction was deemed not related to anthracyclines. Additionally, nine patients had echocardiographic images of insufficient quality to perform reliable measurements. Thereby, a total of 92 patients were included in this study. All patient characteristics at time of ACD diagnosis are outlined in Table 1. Most patients were referred for screening of cardiovascular toxicity $(n=78 ; 85 \%)$ and cardiac screening prior to stem cell transplantation was the main reason for referral $(n=56$; $61 \%$ ). Cardiac complaints, including dyspnea, angina and palpitations were the reason for referral in 14 patients (early $n=5$; late $n=9$ ).

\section{Anthracycline-induced cardiac dysfunction}

Sixty-four patients (70\%) were diagnosed with definite ACD, and thirteen patients (14\%) had ACD with concomitant heart disease(s), including LBBB $(n=3)$, CAD $(n=5)$, moderate mitral valve regurgitation $(n=7)$, noncompaction cardiomyopathy $(n=2)$ and hypertrophic cardiomyopathy $(n=1)$. In 15 patients $(16 \%)$, CAD could not be excluded as additional investigations of coronary artery status had not (yet) been performed. These patients were classified as having possible ACD. 


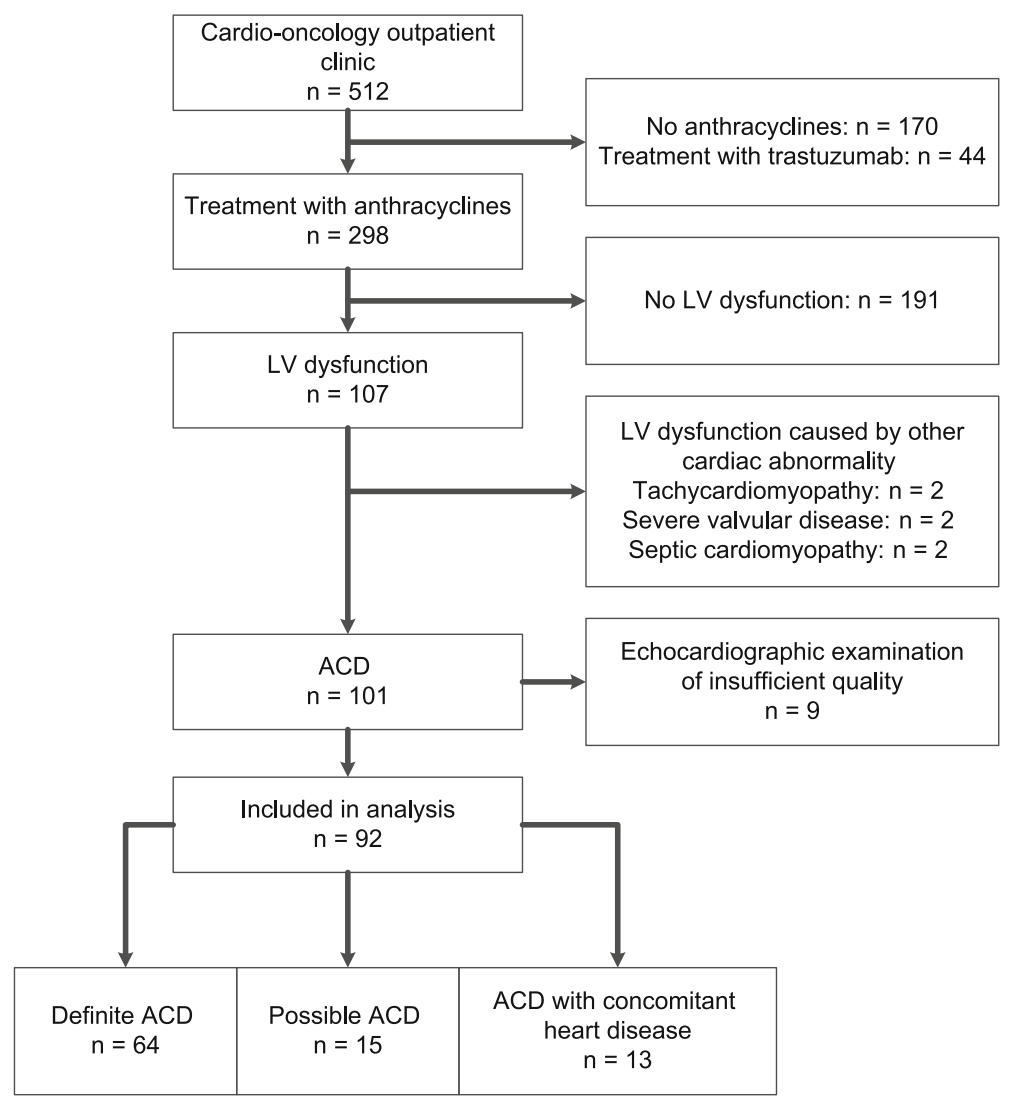

Fig. 1 Flow chart of the study population selection

Of all 92 patients, 32 (35\%) did not have any known cardiovascular risk factors, 43 (47\%) had 1 risk factor, 17 $(19 \%)$ had $\geq 2$ risk factors. Forty-nine patients were diagnosed within 1 year after anthracycline-initiation (median 4 [1.9-6.4] months). ACD was diagnosed late in 43 patients (median 47.7 [41.7-87.3] months) of which 29 patients were diagnosed $>2$ years after initiation of anthracycline-containing anticancer treatment. All patients had finished anthracycline-containing therapy and did not receive further treatment with cardiotoxic agents.

\section{Echocardiographic characterization of ACD at diagnosis}

Overall, the LVEF impairment was mild in 76 patients (83\%), moderate in $12(13 \%)$ and severe in 4 patients (4\%). No echocardiographic differences were found between early- and late diagnosed ACD (Table 2, Fig. 2a). There was no relation between timing of ACD diagnosis and $-\operatorname{LVEF}(\rho=-0.029 p=0.782$; Fig. 3a), - GLS $(\rho=-$ $0.099, p=0.374$; Fig. $3 b)$, or iEDV $(\rho=-0.112 ; p=0.288$; Fig. 3c) at diagnosis. Seventy-three patients (79\%) had a normal indexed end-diastolic volume (iEDV) (Fig. 3c). We found a decreased, normal, and increased LV mass in 2,74 and 15 patients, respectively. Out of the 15 patients with increased LV mass, 7 patients had hypertension and 3 patients had moderate mitral valve regurgitation. No relation between timing of ACD diagnosis and LV geometry was established $(p=0.710)$ (Fig. 3d). There was no correlation between cumulative anthracycline dose and LV mass $(r=0.021, p=0.845)$. Thirty-four patients (38\%) had normal diastolic function, 47 (53\%) had grade I- and 8 (9\%) had grade II diastolic dysfunction.

\section{ACD treatment and clinical follow-up}

The median follow-up time after diagnosis of ACD was 25.0 [10.6-38.2] months (Fig. 2b). HF medication was initiated in 68 patients (74\%) (Table 3). Five of these patients were already on ACE-inhibitors or beta-blockers because of hypertension, and additional HF therapy was given. Intolerance to ACE-inhibitors and betablockers was common, and overall the maximum tolerated dose was low (Table 3). Twenty-four patients (26\%) did not receive HF medication since they had an LVEF of $>45 \%$ and lacked cardiac complaints [11]. Three patients were diagnosed with ACD when they were hospitalized due to $\mathrm{HF}$ and 5 patients were hospitalized with decompensated HF after ACD was diagnosed. No patient was in need for cardiac mechanical support or heart transplantation during follow-up. Implantable cardioverter defibrillator 
Table 1 Characteristics of study participants

\begin{tabular}{|c|c|c|c|c|}
\hline Demographics & Overall $(n=92)$ & Early $(n=49)$ & Late $(n=43)$ & $p$-value \\
\hline Male & $68(74)$ & $38(78)$ & $30(70)$ & \\
\hline Body Mass Index (kg/m²) & $24.5(4.5)$ & $24.8(4.5)$ & $24.1(4.5)$ & 0.422 \\
\hline Body Surface Area $\left(\mathrm{m}^{2}\right)$ & $1.9(0.2)$ & $1.9(0.2)$ & $1.9(0.2)$ & 0.536 \\
\hline Age at start cancer therapy (years) & $48.2(18.1)$ & $52.2(15.8)$ & $43.7(19.2)$ & 0.024 \\
\hline Age at diagnosis ACD (years) & $51.6(16.2)$ & $52.4(16.1)$ & $50.8(16.2)$ & 0.646 \\
\hline \multicolumn{5}{|l|}{ Malignancy } \\
\hline Hematological & $86(94)$ & $48(98)$ & $38(88)$ & \\
\hline Acute leukemia & $44(51)$ & $32(67)$ & $12(32)$ & \\
\hline Non-Hodgkin's lymphoma & $6(7)$ & $1(2)$ & $5(13)$ & \\
\hline Hodgkin's lymphoma & $26(30)$ & $8(17)$ & $18(47)$ & \\
\hline Other $^{\mathrm{a}}$ & $10(12)$ & $7(15)$ & $3(8)$ & \\
\hline Breast cancer & $2(2)$ & $0(0)$ & $2(5)$ & \\
\hline Other solid tumors ${ }^{b}$ & $4(4)$ & $1(2)$ & $3(7)$ & \\
\hline Cumulative anthracycline dose $\left(\mathrm{mg} / \mathrm{m}^{2}\right)$, [IQR] & 329 [200-329] & 329 [180-329] & 308 [200-400] & 0.114 \\
\hline Chest radiation & $7(8)$ & $1(2)$ & $6(14)$ & \\
\hline \multicolumn{5}{|l|}{ Functional class at diagnosis } \\
\hline NYHA class I+ II & $86(94)$ & $47(96)$ & $39(91)$ & \\
\hline NYHA class III + IV & $6(6)$ & $2(4)$ & $4(9)$ & \\
\hline \multicolumn{5}{|l|}{ Electrocardiogram } \\
\hline \multicolumn{5}{|l|}{ Heart rhythm } \\
\hline Sinus rhythm & $90(98)$ & $48(98)$ & $42(98)$ & \\
\hline Atrial fibrillation & $2(2)$ & $1(2)$ & $1(2)$ & \\
\hline \multicolumn{5}{|l|}{ Heart axis } \\
\hline Normal & $82(89)$ & $42(86)$ & $40(93)$ & \\
\hline Left & $8(9)$ & $5(10)$ & $3(7)$ & \\
\hline Right & $2(2)$ & $2(4)$ & $0(0)$ & \\
\hline \multicolumn{5}{|l|}{ Ventricular conduction } \\
\hline Normal & $86(93)$ & $45(92)$ & $41(95)$ & \\
\hline Left bundle branch block & $4(4)$ & $2(4)$ & $2(5)$ & \\
\hline Right bundle branch block & $2(2)$ & $2(4)$ & $0(0)$ & \\
\hline \multicolumn{5}{|l|}{ Cardiovascular risk factors } \\
\hline Hypertension & $19(21)$ & $7(14)$ & $12(28)$ & \\
\hline Diabetes Mellitus & $9(10)$ & $3(6)$ & $6(14)$ & \\
\hline Hyperlipidemia $^{c}$ & $12(17)$ & $5(13)$ & $7(19)$ & \\
\hline Smoking status & $n=80$ & $n=42$ & $n=38$ & \\
\hline Former smoker & $31(39)$ & $24(57)$ & $7(18)$ & \\
\hline Current smoker & $13(16)$ & $6(14)$ & $7(18)$ & \\
\hline Never smoked & $36(45)$ & $12(29)$ & $24(64)$ & \\
\hline Coronary / Peripheral Artery disease & $13(14)$ & $8(17)$ & $5(12)$ & \\
\hline
\end{tabular}

Categorical variables are expressed as $\mathrm{n}(\%)$ and continuous variables as mean (standard deviation) or median [interquartile range]; ${ }^{2}$ Other hematological malignancies included chronic myeloid leukemia $(n=4)$, multiple myeloma $(n=3)$, myelodysplastic syndrome $(n=2)$ and myelofibrosis $(n=1)$; ${ }^{b}$ Other solid tumors included sarcomas $(n=3)$ and Wilms tumor $(n=1) ;{ }^{c} n=75$ patients (38 early ACD; 37 late ACD); ACD Anthracycline-induced cardiac dysfunction 
Table 2 Echocardiographic outcomes of patients with early- and late diagnosed ACD

\begin{tabular}{|c|c|c|c|c|}
\hline & Overall $(n=92)$ & Early $(n=49)$ & Late $(n=43)$ & $p$-value \\
\hline \multicolumn{5}{|l|}{ Left ventricle } \\
\hline IVSd (mm) & $9.7(1.8)$ & $9.8(1.7)$ & $9.6(1.9)$ & 0.543 \\
\hline PWd (mm) & $9.5(1.6)$ & $9.6(1.6)$ & $9.4(1.7)$ & 0.483 \\
\hline LVIDd (mm) & $49.7(6.8)$ & $50.0(7.3)$ & $49.3(6.3)$ & 0.630 \\
\hline iLV mass $\left(\mathrm{g} / \mathrm{m}^{2}\right)$ & $89.9(23.9)$ & $91.3(21.1)$ & $88.4(26.9)$ & 0.572 \\
\hline Increased iLV mass (n) ${ }^{a}$ & $15 / 91(17)$ & $8(17)$ & $7(17)$ & 0.319 \\
\hline $\mathrm{iEDV}\left(\mathrm{mL} / \mathrm{m}^{2}\right)$ & $63.3(15.5)$ & $63.6(14.8)$ & $62.9(16.4)$ & 0.840 \\
\hline Dilated $(n)^{a}$ & $19(21)$ & $9(18)$ & $10(23)$ & 0.334 \\
\hline $\mathrm{iESV}\left(\mathrm{mL} / \mathrm{m}^{2}\right)$ & $36.2(11.2)$ & 35.9 (9.6) & $36.5(13.0)$ & 0.813 \\
\hline Dilated (n) ${ }^{a}$ & $69(75)$ & $40(82)$ & $29(67)$ & 0.178 \\
\hline LVEF (\%) & $43.3(5.5)$ & $43.6(4.9)$ & $43.0(6.2)$ & 0.576 \\
\hline GLS (\%) & $-13.5(3.3)$ & $-13.2(3.1)$ & $-13.7(3.5)$ & 0.550 \\
\hline \multicolumn{5}{|l|}{ Right ventricle } \\
\hline Basal RV dimension (mm) & $32.8(6.2)$ & $32.6(5.4)$ & $33.0(7.1)$ & 0.756 \\
\hline \multicolumn{5}{|l|}{ RV function $(n=91)$} \\
\hline TAPSE (mm) & $19.5(4.6)$ & $19.5(4.3)$ & $19.6(4.9)$ & 0.905 \\
\hline $\mathrm{S}^{\prime}(\mathrm{cm} / \mathrm{sec})$ & $12.1(2.8)$ & $12.2(2.7)$ & $11.9(2.9)$ & 0.632 \\
\hline Impaired (n) ${ }^{a}$ & $14(15)$ & $7(15)$ & $7(16)$ & 0.823 \\
\hline Systolic RV pressure (mmHg) & $21.4(5.6)$ & $21.0(5.2)$ & $21.9(6.1)$ & 0.617 \\
\hline \multicolumn{5}{|l|}{ Diastolic function $(n=89)$} \\
\hline LAVI $\left(\mathrm{mL} / \mathrm{m}^{2}\right)$ & $28.6(9.1)$ & $29.1(8.2)$ & $28.0(9.9)$ & 0.598 \\
\hline $\mathrm{E}(\mathrm{cm} / \mathrm{sec})$ & $55.0(18.5)$ & $54.7(15.80$ & $55.5(21.4)$ & 0.840 \\
\hline $\mathrm{A}(\mathrm{cm} / \mathrm{sec})$ & $63.0(17.0)$ & $63.7(17.3)$ & $62.2(16.9)$ & 0.696 \\
\hline E/A ratio & $0.91(0.39)$ & $0.91(0.40)$ & $0.91(0.39)$ & 0.961 \\
\hline E deceleration time (msec) & $176(51)$ & $182(60)$ & $169(37)$ & 0.271 \\
\hline $\mathrm{E}^{\prime}(\mathrm{cm} / \mathrm{sec})$ & $8.1(2.9)$ & $8.0(3.0)$ & $8.2(2.8)$ & 0.747 \\
\hline$E / E^{\prime}$ & $7.2(2.6)$ & $7.3(2.5)$ & $7.1(2.7)$ & 0.771 \\
\hline
\end{tabular}

Categorical variables are expressed as $\mathrm{n}(\%)$ and continuous variables as mean (standard deviation) or median [interquartile range]. IVSd end-diastolic intraventricular septal dimension, PWd End-diastolic posterior wall dimension, LVEDD Left ventricular end-diastolic dimension, iLV Indexed left ventricular (mass), iEDV Indexed end-diastolic volume, iESV Indexed end-systolic volume, LVEF Left ventricular ejection fraction, GLS Global longitudinal strain, RV Right ventricle, TAPSE Tricuspid annular plain systolic excursion; $S^{\prime}$ Doppler Tissue Imaging-derived S-wave, LAVI Left atrial volume index; ${ }^{a}$ References values of echocardiographic measurements are provided in supplemental Table 1

(ICD) implantation was performed in 4 patients, of which 3 patients also received cardiac resynchronization therapy. One patient was successfully treated with a MitraClip because of severe, secondary mitral regurgitation, which developed after an initial decline in LV function. In total, 38 patients died during follow-up. One patient died due to acute HF. Other deaths were predominantly related to the underlying oncological disease $(n=30)$ or cancer treatment-related infections $(n=2)$. In 5 patients, the cause of death was unknown.

\section{ACD echocardiographic follow-up and reversibility}

Follow-up echocardiographic examinations were available in 67 patients, with a median follow-up time of 17.7 [10.5-26.6] months between diagnosis and the last echocardiographic examination. Of the 53 patients who had a normal iEDV at diagnosis, only 2 patients (3.7\%) developed an iEDV above the upper limit of normal (ULN) during follow-up. Thirteen out of 14 patients who presented with a dilated LV showed normal iEDV values at follow-up. There was no relation between follow-up duration and the change in $\operatorname{iEDV}(\rho=-0.079 ; p=0.524)$.

Sixty-seven patients could be analyzed for reversibility of ACD. Of the 25 patients who were excluded from the analysis, 20 patients died before cardiac follow-up could be performed. In 32 patients (48\%) no recovery of LV function was observed (treatment in $n=24$ ), 10 patients (15\%) had partial recovery (treatment in $n=9$ ), and 25 patients (37\%) showed complete recovery (treatment in $n=17$ ). No differences were observed in GLS at diagnosis between patients with- and without (partial) recovery ((partial) recovery $-13.9 \pm 3.0$; no recovery $-14.0 \pm 3.6$; 


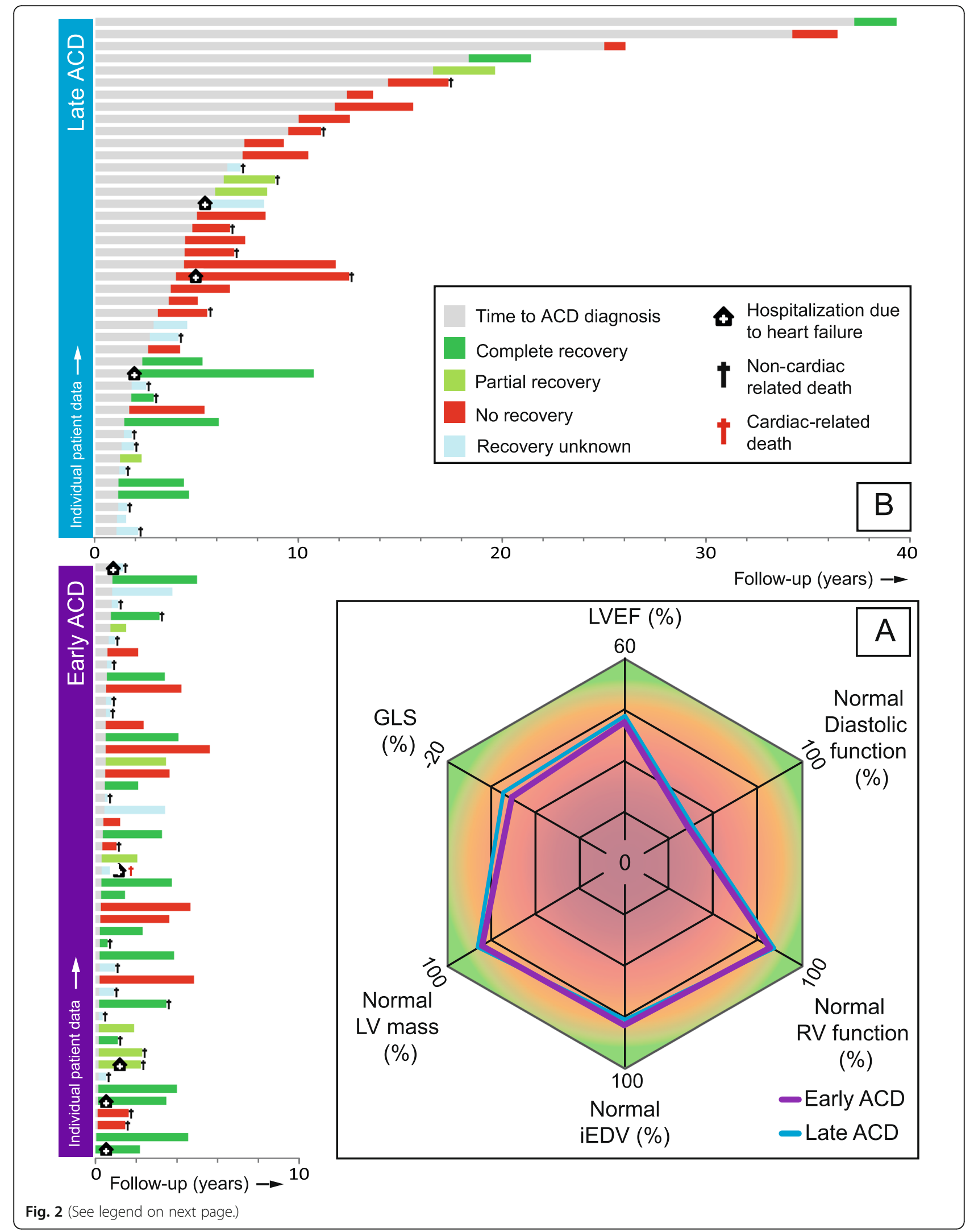


(See figure on previous page.)

Fig. 2 Echocardiographic characterization of early- and late diagnosed anthracycline-induced cardiac dysfunction and follow-up. a The radar chart shows the echocardiographic phenotype at diagnosis of early- and late ACD, which are both characterized by a mild hypokinetic, non-dilated cardiomyopathy. LVEF and GLS are expressed as group means, LV mass, iEDV and RV function are expressed as \% of patients with normal outcomes and diastolic function is expressed as \% of patients with diastolic dysfunction $\leq$ grade ; $\mathbf{b}$ Individual time periods of time to ACD diagnosis and follow-up outcomes regarding i) hospitalization due to heart failure ii) recovery of LV function and iii) (non-)cardiac death

$p=0.908)$. Patients with early ACD were more likely to show (partial) recovery of LV function compared to late ACD patients, without significant differences in maximum tolerated doses of HF medication (Table 3).

\section{Discussion}

The aim of this consecutive cohort study was to evaluate the echocardiographic phenotype of ACD, establish what impact a delayed diagnosis had on the degree of LV dysfunction, LV dimensions and response to HF treatment. The key findings of this study are threefold: (1) the majority of patients presented with mild LV dysfunction without LV dilatation (2) the echocardiographic phenotype was not different in patients diagnosed with early or late ACD and (3) patients with an early ACD diagnosis and prompt initiation of HF treatment were more likely to have a (partial) recovery of LV function, compared to patients with a late ACD diagnosis.

In contrast to previous studies in which ACD is described as a toxic cause of DCM [15], we found that LV dilatation was present in only one-fifth of cases at diagnosis and only two patients developed LV dilatation during a median follow-up of 17.7 months. We believe that there are three major reasons for the absence of a DCMlike phenotype. Firstly, many patients received an early diagnosis of ACD due to serial echocardiographic screening at a cardio-oncology clinic. In the past, the diagnosis of ACD was often established upon the development of symptomatic HF, and subclinical changes in LV function were not detected. In these patients, prolonged activation of compensatory mechanisms, including the renin-angiotensin aldosterone system (RAAS), and subsequent LV remodeling, may have led to a more pronounced dilatation of the LV. However, in our study, dilatation was also not present in the majority of patients with a late ACD diagnosis. The second possible reason for the absence of abnormal dimensions could be related to the reduction of cumulative anthracycline dose over the last decades. While doxorubicin doses exceeding > $500 \mathrm{mg} / \mathrm{m}^{2}$ were commonly administered in the past [1], the maximum cumulative dose of this agent is nowadays restricted to $450 \mathrm{mg} / \mathrm{m}^{2}$ [16], with a median dose of 329 [200-329] $\mathrm{mg} / \mathrm{m} 2$ in this study $(n=9$ receiving $\geq 450$ $\left.\mathrm{mg} / \mathrm{m}^{2}\right)$. It is plausible that these dose restrictions have resulted in an overall milder ACD phenotype. Thirdly, in patients developing LV dysfunction with an LVEF $<45 \%$, HF treatment was promptly initiated, aiming at suppression of RAAS to prevent adverse LV remodeling. The early detection of ACD has shown to be beneficial in one study, where patients with early initiation of therapy were more likely to respond to pharmacological treatment [17]. To our knowledge, our study is the first to validate this time-dependent response to HF treatment. In conclusion, an earlier diagnosis, a restriction in the maximum cumulative anthracycline dose and the initiation of HF treatment might jointly have led to a hypokinetic non-dilated cardiomyopathy rather than DCM [18]. Based upon the results of this study, monitoring of LV function in patients at risk for ACD is recommended to detect subclinical changes in LV function as soon as possible and thereby allow for early initiation of HF treatment in case ACD develops.

We did not find any echocardiographic differences in dimension and function between patients diagnosed early vs. late (Table 2). Furthermore, there were also no discrepancies in LV mass between the two groups. To date, a number of imaging studies have evaluated this parameter in ACD both by CMR [19-21] and echocardiography [22, 23]. Currently, CMR is considered as the gold standard for measurements of cardiac structure and volumes [13]. With this technique, three studies found a decrease in LV mass [19-21], and an inverse correlation with the anthracycline dose [20]. Furthermore, a lower LV mass was predictive of cardiovascular death, appropriate ICD therapy and HF hospitalization in a multivariate model [20]. However, these findings are contradictory to echocardiographic studies which found an increase in LV mass [22, 23]. Armstrong et al. studied adults who were treated with anthracyclines during childhood. They found a reduced LV mass in nearly half of patients [24]. Comparison of CMR with echocardiography performed within $48 \mathrm{~h}$, revealed that echocardiography overestimated LVEF and LV mass and underestimates LV volumes. The absence of reduced LV masses in our study population could therefore be related to LV mass measurements with echocardiography.

In contrast to our hypothesis, our data does not support the progressive nature of ACD regarding cardiac remodeling. To date, longitudinal imaging studies in patients with ACD are scarce, with a small number of studies performed in pediatric [25] and adult cancer patients [22, 26]. Lipshultz et al. prospectively followed 115 survivors of childhood acute leukemia with serial echocardiograms during a median follow-up of 11.8 years 

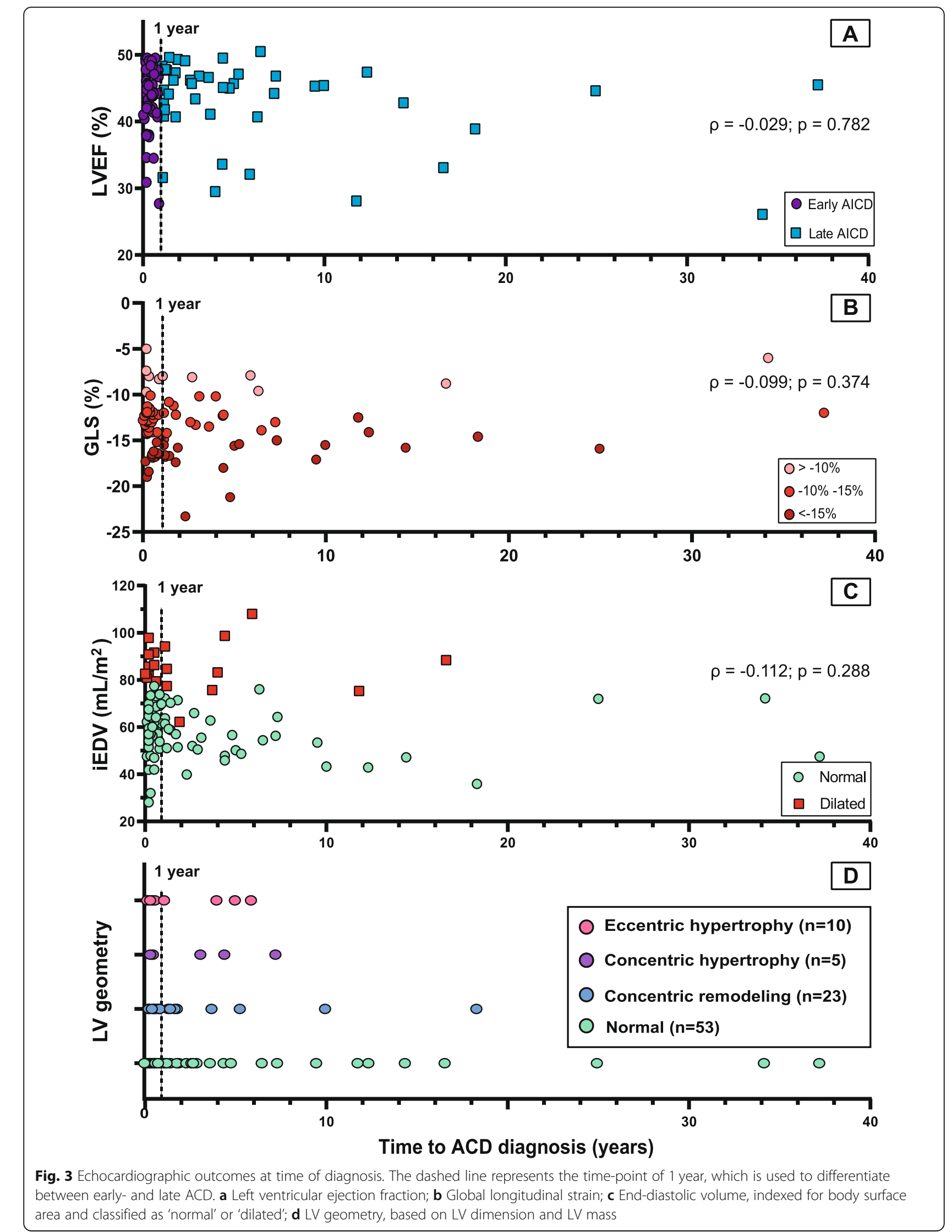
Table 3 Heart failure therapy and response to heart failure treatment

\begin{tabular}{llll}
\hline & Overall $(\boldsymbol{n}=\mathbf{9 2})$ & Early $(\boldsymbol{n}=\mathbf{4 9 )}$ & Late $(\boldsymbol{n}=\mathbf{4 3})$ \\
\hline $\begin{array}{l}\text { Heart failure treatment initiation } \\
\text { Initiated }\end{array}$ & $63(68)$ & $36(73)$ & $27(63)$ \\
Adding to previous therapy & $5(5)$ & $1(2)$ & $4(9)$ \\
LVEF $>45 \%$ and no cardiac complaints & $24(26)$ & $12(24)$ & $12(28)$
\end{tabular}

Number of HF drugs

$\begin{array}{llll}1 & 32(47) & 20(54) & 12(39) \\ 2 & 28(41) & 15(41) & 13(42) \\ 3 & 8(12) & 2(5) & 6(19)\end{array}$

ACE inhibitors / ARB

$\begin{array}{lll}61(90) & 34(92) & 27(87) \\ 25[16.7-50] & 25[16.7-50] & 25[25-41.5]\end{array}$

$$
\text { \% of target dose }
$$

\section{Beta-blockers}

n

$\%$ of target dose

MRA

$$
\text { \% of target dose }
$$

\section{Side effects}

$$
\begin{aligned}
& \text { Hypotension } \\
& \text { Renal dysfunction } \\
& \text { Other }
\end{aligned}
$$

Duration of HF treatment (months)

Response to HF therapy

\section{Response to HF therapy}

Response to HF therapy

Early

Number of HF drugs

1
2
3

ACE inhibitors / ARB

$$
\text { \% of target dose }
$$

\section{8 (49) \\ 18.75 [12.5-25]}

$$
\begin{aligned}
& 6(9) \\
& 37.5 \text { [25-50] }
\end{aligned}
$$$$
2 \text { (5) }
$$$$
37.5 \text { [25-50] }
$$

$10(27)$

1 (3)

3 (8)

14.6 [3.4-28.0]

$18(26)$
$3(4)$
$6(9)$
$16.8[4.8-29.8]$

\section{Overall}

$(n=50)$

(partial) recovery $(n=26)$

No recovery $(n=24)$

p-value

$26(51)$
$24(49)$
$22(44)$
$20(40)$
$8(16)$

18 (69)

8 (31)

$12(46)$

9 (35)

5 (19)

46 (92)

25 [16.7-50]

$23(88)$
$25[12.5-50]$

21 (68)

25 [12.5-50]

4 (13)

37.5 [25-50]

$8(26)$
$2(6)$
$3(10)$
$25.3[10.1-35.2]$

$0.038^{*}$

$$
\begin{array}{lll}
29(59) & 16(62) & 14(58) \\
25[12.5-50] & 25[12.5-50] & 37.5[12.5-50] \\
21.9[13.5-30.9] & 22.3[13.5-30.9] & 25.9[13.3-36.6]
\end{array}
$$

$16(67)$$$
3(12)
$$$$
23 \text { (96) }
$$$$
25 \text { [25-50] }
$$

Beta-blockers

$$
\text { \% of target dose }
$$

Duration of HF treatment (months)

Target doses are expressed as percentage of target dose according to the ESC Guideline on Heart Failure [12]. Categorical variables are expressed as $\mathrm{n}$ (\%) and continuous variables as mean (standard deviation) or median [interquartile range]. LVEF Left ventricular ejection fraction, $H F$ Heart failure, $A C E$ Angiotensin converting enzyme, ARB Angiotensin II receptor blockers, MRA Mineralocorticoid receptor antagonist; * $p$-value $<0.05$

[25]. The LV contractility initially declined after doxorubicin containing chemotherapy, normalized the next 6 years and subsequently became significantly depressed $>$
12 years after the cancer diagnosis. In another prospective cohort study among 277 breast cancer patients treated with doxorubicin (36\% in combination with 
trastuzumab) the LVEF decline was not progressive during a median follow-up of 2 years [22]. In a study by Jones et al. in 143 patients that were followed for 2 years, none transitioned to more advanced HF stages [26]. In our study only $5.5 \%$ of patients progressed to symptomatic HF. Nevertheless, the presence of asymptomatic LV dysfunction gives an increased risk of ultimately progressing to symptomatic HF. In a meta-analysis evaluating the risk in patients with systolic LV dysfunction due to various etiologies, the incidence of symptomatic HF was 8.4 (95\% CI 4.0-12.8) per 100 person-years, compared to 1.04 (95\% CI $0.0-2.2)$ per 100 person-years in the absence of LV dysfunction, equaling a relative risk of 4.6 (95\% CI 2.2-9.8) [27]. This meta-analysis illustrates the importance of implementing effective strategies in the pre-symptomatic stages to mitigate the progression rate to symptomatic HF. This progression might also be dependent on the development of other cardiac stressors, such as hypertension, valvular disease, $\mathrm{CAD}$, or the presence of pathogenic variants in cardiomyopathy-associated genes [28, 29]. In the absence of these so called "second-hits", it is possible that a considerable proportion of patients only develops mild LV dysfunction after anthracycline-containing chemotherapy, that remains stable for years. Larger observational cohort studies, preferably with long-term followup can shed more light on the natural course of this specific disease entity. Also, the outcomes of the TITANstudy, which compares intensive cardiac monitoring and -treatment to usual care, will be informative on the added value of early identification of ACD [30].

\section{Limitations}

Our analysis was restricted to patients that visited the cardio-oncology outpatient clinic. Patients treated with potentially cardiotoxic cancer therapy prior to its launch currently receive cardiological follow-up to screen for long-term cardiovascular complications. It is possible that patients who developed a more severe ACD phenotype presented at the emergency care unit earlier and were never seen in an outpatient clinic setting and therefore were not included in our study. Also, patients deemed to be at low risk for ACD do not receive cardiac screening per protocol at our cardio-oncology service. This may overall lead to an underestimation of ACD in the population of cancer patients treated with anthracyclines.

Furthermore, the current study used patient information collected during standard clinical care. Albeit the follow-up of these patients is standardized to a great extent [11], there still was heterogeneity in the data. Even though current guidelines recommend to perform cardiac screening prior to treatment [31], many patients were referred after initiation of anthracycline-treatment, and therefore lacked cardiac baseline assessment. Subtle changes in LV dimensions and -volumes within patients could therefore have been overlooked. In addition, for patients that did not undergo an echocardiography at baseline, pre-existent impaired cardiac function could have been misclassified as ACD. Also, additional testing for other causes of impaired LV function, such as CAD, had not (yet) been performed in a number of patients, leaving uncertainty of the diagnosis of ACD. Nevertheless, this represents only a small subset of our cohort where, from a clinical point of view, ACD was very likely.

Biomarkers, such as troponin or BNP, are not routinely performed at our cardio-oncology clinic and were only available in a limited number of patients. We therefore were unable to include the outcomes of biomarkers in our analysis.

\section{Conclusions}

We found that the ACD phenotype overall was mild and a majority of patients lacked cardiac complaints. In the absence of serial echocardiographic assessment, it therefore is plausible that the impaired cardiac function would have remained undetected. Since response to HF treatment is time-dependent, detection of asymptomatic LV dysfunction is of great importance. When cardiac compromise is detected, other cardiovascular risk factors can be treated more aggressively, potentially decreasing the risk of patients progressing to more advanced HF stages. Moreover, if a patient is planned to receive further cardiotoxic treatment, preventive actions can be considered [31]. The involvement of a cardiologist in a multidisciplinary setting at cardio-oncology clinics is valuable to allow for the early detection of ACD and other adverse cardiovascular effects during cardiotoxic treatment [32].

Importantly, future research within the field of cardio-oncology should not only focus on the added value of cardiac screening, but also possible drawbacks including medicalization and increased healthcare costs [33]. Trials evaluating different follow-up strategies, such as the TITAN-study [30], are required to achieve an optimal risk-benefit ratio. It is plausible that the optimal strategy varies per cancer type, as often the prognosis of the malignancy is a dominant factor. Longitudinal cohort studies establishing more insight into the natural course of ACD are herein of pivotal importance.

\section{Supplementary information}

Supplementary information accompanies this paper at https://doi.org/10. 1186/s40959-020-00079-3.

Additional file 1: Supplemental Table 1. Reference values of echocardiographic measurements and international guidelines on echocardiographic examination [16]. 


\section{Abbreviations}

ACD: Anthracycline-induced cardiac dysfunction; DCM: Dilated cardiomyopathy; LV: Left ventricle; LVEF: Left ventricular ejection fraction; iEDV: Indexed end-diastolic volume; CTRCD: Cancer therapy-related cardiac dysfunction; CMR: Cardiac Magnetic resonance; MUGA: Multiple-gated acquisition; ESC: European Society of Cardiology; LBBB: Left bundle branch block; CAD: Coronary artery disease; CT: Computed tomography; BSA: Body surface area; GLS: Global longitudinal strain; LVIDd: Left ventricular internal diameter at end-diastole; RWT: Relative wall thickness; TAPSE: Tricuspid annular plain systolic excursion; DTI: Doppler tissue imaging; EACVI: European Association of Cardiovascular Imaging; LLN: Lower limit of normal; IQR: Interquartile range; SD: Standard deviation; ANOVA: Analysis of variance; NYHA: New York Heart Association; RV: Right ventricle; ACE: Angiotensin converting enzyme; ICD: Implantable cardiac defibrillator; ULN: Upper limit of normal; RAAS: Renin-angiotensin aldosterone system

\section{Acknowledgements}

Not applicable.

\section{Authors' contributions}

JAMK, ML and AJT contributed to data collection, study design, data analysis, data interpretation, and writing of the manuscript. MJC, PAD and FWA contributed to writing the manuscript. All authors critically reviewed the manuscript and provided important intellectual contributions. The final manuscript was approved by all authors.

\section{Funding}

Dr. Asselbergs has reported support from the University College London Hospitals, National Institute for Health Research Biomedical Research Centre. Dr. Linschoten has reported support from the Alexandre Suerman Stipend of the University Medical Center (UMC) Utrecht. All other authors have reported that they have no relationships relevant to the contents of this paper to disclose.

\section{Availability of data and materials}

The datasets generated and analysed during the current study are not publicly available due to protecting participant confidentiality but are available from the corresponding author on reasonable request.

\section{Ethics approval and consent to participate}

The study was exempted from formal approval by the Medical Ethics Committee (METC 19-304/C,Utrecht, the Netherlands).

\section{Consent for publication}

Not applicable.

\section{Competing interests}

None declared.

\section{Author details}

'Department of Cardiology, Division of Heart and Lungs, University Medical Center Utrecht, University of Utrecht, E03.511, PO Box 85500, 3508 GA Utrecht, The Netherlands. ${ }^{2}$ Netherlands Heart Institute, Utrecht, The Netherlands. ${ }^{3}$ Central Military Hospital, Utrecht, The Netherlands. ${ }^{4}$ Health Data Research UK and Institute of Health Informatics, University College London, London, UK. ${ }^{5}$ Institute of Cardiovascular Science, Faculty of Population Health Sciences, University College London, London, UK.

\section{Received: 2 August 2020 Accepted: 2 October 2020}

\section{Published online: 13 October 2020}

\section{References}

1. Von Hoff DD, Layard MW, Basa P, Davis HL, von Hoff AL, Rozencweig M, et al. Risk factors for doxorubicin-induced congestive heart failure. Ann Intern Med. 1979;91:710-7. https://doi.org/10.7326/0003-4819-91-5-710.

2. Lotrionte M, Biondi-Zoccai G, Abbate A, Lanzetta G, D'Ascenzo F, Malavasi V, et al. Review and meta-analysis of incidence and clinical predictors of anthracycline cardiotoxicity. Am J Cardiol. 2013;112:1980-4. https://doi.org/ 10.1016/j.amjcard.2013.08.026

3. Fornaro A, Olivotto I, Rigacci L, Ciaccheri M, Tomberli B, Ferrantini C, et al, Comparison of long-term outcome in anthracycline-related versus idiopathic dilated cardiomyopathy: a single Centre experience. Eur J Heart Fail. 2018;20(5):898-906. https://doi.org/10.1002/ejhf.1049.

4. Felker GM, Thompson RE, Hare JM, Hruban RH, Clemetson DE, Howard DL, et al. Underlying causes and long-term survival in patients with initially unexplained cardiomyopathy. N Engl J Med. 2000;342(15):1077-84. https:// doi.org/10.1056/NEJM200004133421502.

5. Zamorano JL, Lancellotti P, Rodriguez Muñoz D, Aboyans V, Asteggiano R, Galderisi M, et al. 2016 ESC position paper on cancer treatments and cardiovascular toxicity developed under the auspices of the ESC Committee for practice guidelines: the task force for cancer treatments and cardiovascular toxicity of the European Society of Cardiology (ESC). Eur Heart J. 2016;37(36):2768-801. https://doi.org/10.1093/eurheartj/ehw211.

6. Cardinale D, Sandri MT, Colombo A, Colombo N, Boeri M, Lamantia G, et al. Prognostic value of troponin I in cardiac risk stratification of cancer patients undergoing high-dose chemotherapy. Circulation. 2004;109(22):2749-54. https://doi.org/10.1161/01.CIR.0000130926.51766.CC.

7. Auner HW, Tinchon $\mathrm{C}$, Linkesch $\mathrm{W}$, Tiran $\mathrm{A}$, Quehenberger $\mathrm{F}$, Link $\mathrm{H}$, et al. Prolonged monitoring of troponin $\mathrm{T}$ for the detection of anthracycline cardiotoxicity in adults with hematological malignancies. Ann Hematol. 2003;82(4):218-22. https://doi.org/10.1007/s00277-003-0615-3.

8. Cardinale D, Colombo A, Bacchiani G, Tedeschi I, Meroni CA, Veglia F, et al. Early detection of Anthracycline Cardiotoxicity and improvement with heart failure therapy. Circulation. 2015;131(22):1981-8. https://doi.org/10.1161/ CIRCULATIONAHA.114.013777.

9. Mann DL, Bristow MR. Mechanisms and models in heart failure: the biomechanical model and beyond. Circulation. 2005;111:2837-49. https:// doi.org/10.1161/CIRCULATIONAHA.104.500546.

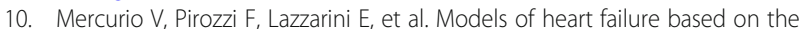
cardiotoxicity of anticancer drugs. J Card Fail. 2016;22(6):449-58. https://doi. org/10.1016/j.cardfail.2016.04.008.

11. Teske AJ, Linschoten M, Kamphuis JAM, Naaktgeboren WR, Leiner T, van de

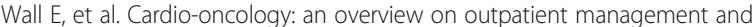
future developments. Neth Hear J. 2018;26(11):521-32. https://doi.org/10. 1007/s12471-018-1148-7.

12. Ponikowski P, Voors AA, Anker SD, Bueno H, Cleland JG, Coats AJ, et al. 2016 ESC guidelines for the diagnosis and treatment of acute and chronic heart failure: the task force for the diagnosis and treatment of acute and chronic heart failure of the European Society of Cardiology (ESC). Developed with the special contribution of the heart failure association (HFA) of the ESC. Eur J Heart Fail. 2016;18(8):891-975. https://doi.org/10.1093/eurheartj/ehw128.

13. Plana JC, Galderisi M, Barac A, Ewer MS, Ky B, Scherrer-Crosbie M, et al. Expert consensus for multimodality imaging evaluation of adult patients during and after Cancer therapy: a report from the American Society of Echocardiography and the European Association of Cardiovascular Imaging. Eur Heart J Cardiovasc Imaging. 2014;15(10):1063-93. https://doi.org/10. 1016/j.echo.2014.07.012.

14. Shankar SM, Marina N, Hudson MM, Hodgson DC, Adams J, Landier W, et al. Monitoring for cardiovascular disease in survivors of childhood Cancer: report from the cardiovascular disease task force of the Children's oncology group. Pediatrics. 2008;121(2):e387-96. https://doi.org/10.1542/peds.2007-0575.

15. Japp AG, Gulati A, Cook SA, Cowie MR, Prasad SK. The diagnosis and evaluation of dilated cardiomyopathy. J Am Coll Cardiol. 2016;67(25):29963010. https://doi.org/10.1016/j.jacc.2016.03.590.

16. Ewer MS, Ewer SM. Cardiotoxicity of anticancer treatments. Nat Rev Cardiol. 2015;12(9):547-58. https://doi.org/10.1038/nrcardio.2015.65.

17. Cardinale D, Colombo A, Lamantia G, Colombo N, Civelli M, De Giacomi G, et al. Anthracycline-induced cardiomyopathy: clinical relevance and response to pharmacologic therapy. J Am Coll Cardiol. 2010;55(3):213-20. https://doi.org/10.1016/j.jacc.2009.03.095.

18. Pinto YM, Elliott PM, Arbustini E, Adler Y, Anastasakis A, Böhm M, et al. Proposal for a revised definition of dilated cardiomyopathy, hypokinetic non-dilated cardiomyopathy, and its implications for clinical practice: a position statement of the ESC working group on myocardial and pericardial diseases. Eur Heart J. 2016:37(23):1850-8. https://doi.org/10.1093/eurheartj/ ehv727.

19. Ferreira de Souza T, Quinaglia AC, Silva T, Osorio Costa F, Sha R, Neilan TG Velloso L, et al. Anthracycline therapy is associated with Cardiomyocyte atrophy and preclinical manifestations of heart disease. JACC CardiovasC Imaging. 2018;11(8):1045-55. https://doi.org/10.1016/j.jcmg.2018.05.012.

20. Neilan TG, Coelho-Filho OR, Pena-Herrera D, Shah RV, Jerosch-Herold M, Francis SA, et al. Left ventricular mass in patients with a cardiomyopathy 
after treatment with Anthracyclines. Am J Cardiol. 2012;110(11):1679-86. https://doi.org/10.1016/j.amjcard.2012.07.040.

21. Jordan JH, Castellino SM, Meléndez GC, Klepin HD, Ellis LR, Lamar Z, et al. Left ventricular mass change after Anthracycline chemotherapy. Circ Heart Fail. 2018;11(7):e004560. https://doi.org/10.1161/CIRCHEARTFAlLURE.117. 004560.

22. Narayan HK, Finkelman B, French B, Plappert T, Hyman D, Smith AM, et al. Detailed echocardiographic Phenotyping in breast Cancer patients: associations with ejection fraction decline, recovery, and heart failure symptoms over 3 years of follow-up. Circulation. 2017;135(15):1397-412. https://doi.org/10.1161/CIRCULATIONAHA.116.023463.

23. Tan TC, Bouras S, Sawaya H, Sebag IA, Cohen V, Picard MH, et al. Time trends of left ventricular ejection fraction and myocardial deformation indices in a cohort of women with breast Cancer treated with Anthracyclines, Taxanes, and Trastuzumab. J Am Soc Echocardiogr. 2015; 28(5):509-14. https://doi.org/10.1016/j.echo.2015.02.001

24. Armstrong GT, Plana JC, Zhang N, Srivastava D, Green DM, Ness KK, et al. Screening adult survivors of childhood Cancer for cardiomyopathy: comparison of echocardiography and cardiac magnetic resonance imaging. J Clin Oncol. 2012;30(23):2876-84. https://doi.org/10.1200/JCO.2011.40.3584.

25. Lipshultz SE, Lipsitz SR, Sallan SE, Dalton VM, Mone SM, Gelber RD, et al. Chronic progressive cardiac dysfunction years after doxorubicin therapy for childhood acute lymphoblastic leukemia. J Clin Oncol. 2005;23(12):2629-36. https://doi.org/10.1200/JCO.2005.12.121.

26. Jones DN, Jordan JH, Meléndez GC, Lamar Z, Thomas A, Kitzman DW, et al. Frequency of transition from stage a to stage $B$ heart failure after initiating potentially Cardiotoxic chemotherapy. JACC Heart Fail. 2018;6(12):1023-32. https://doi.org/10.1016/j.jchf.2018.08.005.

27. Echouffo-Tcheugui JB, Ergou S, Butler J, Yancy CW, Fonarow GC. Assessing the risk of progression from asymptomatic left ventricular dysfunction to overt heart failure: a systematic overview and meta-analysis. JACC Heart Fail. 2016;4(4):237-48. https://doi.org/10.1016/j.jchf.2015.09.015.

28. Linschoten M, Teske AJ, Cramer MJ, van der Wall E, Asselbergs FW. Chemotherapy-related cardiac dysfunction: a systematic review of genetic variants modulating individual risk. Circ Genom Precis Med. 2018;11(1): e001753. https://doi.org/10.1161/CIRCGEN.117.001753.

29. Garcia-Pavia P, Kim Y, Restrepo-Cordoba MA, Lunde IG, Wakimoto H, Smith $\mathrm{AM}$, et al. Genetic variants associated with Cancer therapy-induced cardiomyopathy. Circulation. 2019;140(1):31-41. https://doi.org/10.1161/ CIRCULATIONAHA.118.037934.

30. Pituskin E, Haykowsky M, McNeely M, Mackey J, Chua N, Paterson I. Rationale and design of the multidisciplinary team IntervenTion in cArdiooNcology study (TITAN). BMC Cancer. 2016;16:733.

31. Curigliano G, Lenihan D, Fradley M, Ganatra S, Barac A, Blaes A, et al. Management of cardiac disease in cancer patients throughout oncological treatment: ESMO consensus recommendations. Ann Oncol. 2020;31(2):17190. https://doi.org/10.1016/j.annonc.2019.10.023.

32. Ammon M, Arenja N, Leibundqut G, Buechel RR, Kuster GM, Kaufmann BA et al. Cardiovascular management of cancer patients with chemotherapyassociated left ventricular systolic dysfunction in real-world clinical practice J Card Fail. 2013;19(9):629-34. https://doi.org/10.1016/j.cardfail.2013.07.007.

33. Levis BE, Binkley PF, Shapiro CL. Cardiotoxic effects of anthracycline-based therapy: what is the evidence and what are the potential harms? Lancet Oncol. 2017;18(8):e445-56. https://doi.org/10.1016/S1470-2045(17)30535-1.

\section{Publisher's Note}

Springer Nature remains neutral with regard to jurisdictional claims in published maps and institutional affiliations.

Ready to submit your research? Choose BMC and benefit from:
- fast, convenient online submission
- thorough peer review by experienced researchers in your field
- rapid publication on acceptance
- support for research data, including large and complex data types
- gold Open Access which fosters wider collaboration and increased citations
- maximum visibility for your research: over 100M website views per year
At BMC, research is always in progress.
Learn more biomedcentral.com/submissions

